\title{
Single-spin Azimuthal Asymmetries in Electroproduction of Neutral Pions in Semi-inclusive Deep-inelastic Scattering
}

A. Airapetian, ${ }^{31}$ N. Akopov, ${ }^{31}$ Z. Akopov ${ }^{31}$ M. Amarian, ${ }^{26,31}$ E.C. Aschenauer, ${ }^{7}$ H. Avakian, ${ }^{11}$ R. Avakian, ${ }^{31}$ A. Avetissian, ${ }^{31}$ E. Avetissian, ${ }^{31}$ P. Bailey, ${ }^{15}$ B. Bains,${ }^{15}$ V. Baturin,${ }^{24}$ C. Baumgarten, ${ }^{21}$ M. Beckmann,${ }^{6,12}$ S. Belostotski, ${ }^{24}$ S. Bernreuther, ${ }^{29}$ N. Bianchi, ${ }^{11}$ H. Böttcher, ${ }^{7}$ A. Borissov,${ }^{6,19}$ O. Bouhali, ${ }^{23}$ M. Bouwhuis, ${ }^{15}$ J. Brack, ${ }^{5}$ S. Brauksiepe, ${ }^{12}$ W. Brückner, ${ }^{14}$ A. Brüll,,${ }^{18}$ I. Brunn, ${ }^{9}$ H.J. Bulten, ${ }^{23,30}$ G.P. Capitani, ${ }^{11}$ P. Chumney, ${ }^{22}$ E. Cisbani, ${ }^{26}$ G. Ciullo, ${ }^{10}$ G.R. Court,${ }^{16}$ P.F. Dalpiaz,${ }^{10}$ R. De Leo, ${ }^{3}$ L. De Nardo,${ }^{1}$ E. De Sanctis,${ }^{11}$ D. De Schepper, ${ }^{2}$ E. Devitsin, ${ }^{20}$ P.K.A. de Witt Huberts, ${ }^{23}$ P. Di Nezza,,${ }^{11}$ V. Djordjadze,${ }^{7}$ M. Düren, ${ }^{9}$ M. Ehrenfried, ${ }^{7}$ G. Elbakian, ${ }^{31}$ F. Ellinghaus,${ }^{7}$ J. Ely, ${ }^{5}$ A. Fantoni, ${ }^{11}$ A. Fechtchenko, ${ }^{8}$ L. Felawka,${ }^{28}$ B.W. Filippone,${ }^{4}$ H. Fischer, ${ }^{12}$ B. Fox,${ }^{5}$ J. Franz,${ }^{12}$ S. Frullani, ${ }^{26}$ Y. Gärber,${ }^{7,9}$ F. Garibaldi,${ }^{26}$ E. Garutti ${ }^{23}$ G. Gavrilov, ${ }^{24}$ V. Gharibyan, ${ }^{31}$ A. Golendukhin, ${ }^{6,21,31}$ G. Graw,${ }^{21}$ O. Grebeniouk, ${ }^{24}$ P.W. Green, ${ }^{1,28}$ L.G. Greeniaus, ${ }^{1,28}$ A. Gute, ${ }^{9}$ W. Haeberli, ${ }^{17}$ K. Hafidi, ${ }^{2}$ M. Hartig, ${ }^{28}$ D. Hasch,${ }^{9}{ }^{11}$ D. Heesbeen,${ }^{23}$ F.H. Heinsius, ${ }^{12}$ M. Henoch, ${ }^{9}$ R. Hertenberger, ${ }^{21}$ W.H.A. Hesselink,${ }^{23,30}$ G. Hofman, ${ }^{5}$ Y. Holler ${ }^{6}$ R.J. Holt,${ }^{2,15}$ B. Hommez ${ }^{13}$ G. Iarygin,${ }^{8}$ A. Izotov, ${ }^{24}$ H.E. Jackson, ${ }^{2}$ A. Jgoun,${ }^{24}$ P. Jung, ${ }^{7}$ R. Kaiser,${ }^{7}$ J. Kanesaka, ${ }^{29}$ E. Kinney,${ }^{5}$ A. Kisselev, ${ }^{2,24}$ P. Kitching, ${ }^{1}$ H. Kobayashi, ${ }^{29}$ N. Koch, ${ }^{9}$ K. Königsmann,${ }^{12}$ H. Kolster, ${ }^{18,23}$ V. Korotkov, ${ }^{7}$ E. Kotik, ${ }^{1}$ V. Kozlov, ${ }^{20}$ B. Krauss,${ }^{9}$ V.G. Krivokhijine, ${ }^{8}$ G. Kyle,${ }^{22}$ L. Lagamba,${ }^{3}$ A. Laziev, ${ }^{23,30}$ P. Lenisa, ${ }^{10}$ P. Liebing, ${ }^{7}$ T. Lindemann, ${ }^{6}$ W. Lorenzon, ${ }^{19}$ A. Maas,${ }^{7}$ N.C.R. Makins,${ }^{15}$ H. Marukyan,${ }^{31}$ F. Masoli,${ }^{10}$ M. McAndrew ${ }^{16}$ K. McIlhany, ${ }^{4,18}$ F. Meissner, ${ }^{21}$ F. Menden, ${ }^{12}$ N. Meyners, ${ }^{6}$ O. Mikloukho, ${ }^{24}$ C.A. Miller, ${ }^{1,28}$ R. Milner, ${ }^{18}$ V. Muccifora,${ }^{11}$ R. Mussa,,${ }^{10}$ A. Nagaitsev,${ }^{8}$ E. Nappi,${ }^{3}$ Y. Naryshkin,${ }^{24}$ A. Nass,${ }^{9}$ K. Negodaeva, ${ }^{7}$

W.-D. Nowak, ${ }^{7}$ K. Oganessyan, ${ }^{6,11}$ T.G. O'Neill, ${ }^{2}$ B.R. Owen, ${ }^{15}$ S.F. Pate,${ }^{22}$ S. Potashov, ${ }^{20}$ D.H. Potterveld,${ }^{2}$ M. Raithel,${ }^{9}$ G. Rakness,${ }^{5}$ V. Rappoport,${ }^{24}$ R. Redwine, ${ }^{18}$ D. Reggiani, ${ }^{10}$ A.R. Reolon, ${ }^{11}$ K. Rith,${ }^{9}$ D. Robinson,${ }^{15}$ A. Rostomyan, ${ }^{31}$ M. Ruh, ${ }^{12}$ D. Ryckbosch,,${ }^{13}$ Y. Sakemi ${ }^{29}$ I. Sanjiev ${ }^{2,24}$ F. Sato, ${ }^{29}$ I. Savin, ${ }^{8}$ C. Scarlett, ${ }^{19}$ A. Schäfer, ${ }^{25}$ C. Schill, ${ }^{12}$ F. Schmidt, ${ }^{9}$ G. Schnell,,${ }^{7,22}$ K.P. Schüler, ${ }^{6}$ A. Schwind, ${ }^{7}$ J. Seibert, ${ }^{12}$ B. Seitz, ${ }^{1}$ T.-A. Shibata, ${ }^{29}$ V. Shutov ${ }^{8}$ M.C. Simani, ${ }^{23,30}$ A. Simon, ${ }^{12}$ K. Sinram, ${ }^{6}$ E. Steffens, ${ }^{9}$ J.J.M. Steijger, ${ }^{23}$ J. Stewart, ${ }^{2,7,16,28}$ U. Stösslein, ${ }^{5,7}$ K. Suetsugu, ${ }^{29}$ S. Taroian, ${ }^{31}$ A. Terkulov, ${ }^{20}$ O. Teryaev,${ }^{8,25}$ S. Tessarin, ${ }^{10}$ E. Thomas, ${ }^{11}$ B. Tipton,${ }^{4}$ M. Tytgat,,${ }^{13}$ G.M. Urciuoli, ${ }^{26}$ J.F.J. van den Brand, ${ }^{23,30}$ G. van der Steenhoven, ${ }^{23}$ R. van de Vyver, ${ }^{13}$ J.J. van Hunen, ${ }^{23}$ M.C. Vetterli, ${ }^{27,28}$ V. Vikhrov,${ }^{24}$ M.G. Vincter, ${ }^{1}$ J. Visser,${ }^{23}$ C. Weiskopf,${ }^{9}$ J. Wendland, ${ }^{27,28}$ J. Wilbert, ${ }^{9}$ T. Wise, ${ }^{17}$ S. Yen ${ }^{28}$ S. Yoneyama, ${ }^{29}$ and H. Zohrabian ${ }^{31}$

(The HERMES Collaboration)

\footnotetext{
${ }^{1}$ Department of Physics, University of Alberta, Edmonton, Alberta T6G 2J1, Canada

${ }^{2}$ Physics Division, Argonne National Laboratory, Argonne, Illinois 60439-4843, USA

${ }^{3}$ Istituto Nazionale di Fisica Nucleare, Sezione di Bari, 70124 Bari, Italy

${ }^{4}$ W.K. Kellogg Radiation Laboratory, California Institute of Technology, Pasadena, California 91125, USA

${ }^{5}$ Nuclear Physics Laboratory, University of Colorado, Boulder, Colorado 80309-0446, USA

${ }^{6}$ DESY, Deutsches Elektronen Synchrotron, 22603 Hamburg, Germany

${ }^{7}$ DESY Zeuthen, 15738 Zeuthen, Germany

${ }^{8}$ Joint Institute for Nuclear Research, 141980 Dubna, Russia

${ }^{9}$ Physikalisches Institut, Universität Erlangen-Nürnberg, 91058 Erlangen, Germany

${ }^{10}$ Istituto Nazionale di Fisica Nucleare, Sezione di Ferrara and Dipartimento di Fisica, Università di Ferrara, 44100 Ferrara, Italy

${ }^{11}$ Istituto Nazionale di Fisica Nucleare, Laboratori Nazionali di Frascati, 00044 Frascati, Italy

${ }^{12}$ Fakultät für Physik, Universität Freiburg, 79104 Freiburg, Germany

${ }^{13}$ Department of Subatomic and Radiation Physics, University of Gent, 9000 Gent, Belgium

${ }^{14}$ Max-Planck-Institut für Kernphysik, 69029 Heidelberg, Germany

${ }^{15}$ Department of Physics, University of Illinois, Urbana, Illinois 61801, USA

${ }^{16}$ Physics Department, University of Liverpool, Liverpool L69 7ZE, United Kingdom

${ }^{17}$ Department of Physics, University of Wisconsin-Madison, Madison, Wisconsin 53706, USA

${ }^{18}$ Laboratory for Nuclear Science, Massachusetts Institute of Technology, Cambridge, Massachusetts 02139, USA

${ }^{19}$ Randall Laboratory of Physics, University of Michigan, Ann Arbor, Michigan 48109-1120, USA

${ }^{20}$ Lebedev Physical Institute, 117924 Moscow, Russia

${ }^{21}$ Sektion Physik, Universität München, 85748 Garching, Germany

${ }^{22}$ Department of Physics, New Mexico State University, Las Cruces, New Mexico 88003, USA

${ }^{23}$ Nationaal Instituut voor Kernfysica en Hoge-Energiefysica (NIKHEF), 1009 DB Amsterdam, The Netherlands

${ }^{24}$ Petersburg Nuclear Physics Institute, St. Petersburg, Gatchina, 188350 Russia

${ }^{25}$ Institut für Theoretische Physik, Universität Regensburg, 93040 Regensburg, Germany

${ }^{26}$ Istituto Nazionale di Fisica Nucleare, Sezione Sanità and Physics Laboratory, Istituto Superiore di Sanità, 00161 Roma,
} 


\author{
Italy \\ ${ }^{27}$ Department of Physics, Simon Fraser University, Burnaby, British Columbia V5A 1S6, Canada \\ ${ }^{28}$ TRIUMF, Vancouver, British Columbia V6T 2A3, Canada \\ ${ }^{29}$ Department of Physics, Tokyo Institute of Technology, Tokyo 152, Japan \\ ${ }^{30}$ Department of Physics and Astronomy, Vrije Universiteit, $1081 \mathrm{HV}$ Amsterdam, The Netherlands \\ ${ }^{31}$ Yerevan Physics Institute, 375036, Yerevan, Armenia
}

\begin{abstract}
A single-spin asymmetry in the azimuthal distribution of neutral pions relative to the lepton scattering plane has been measured for the first time in deep-inelastic scattering of positrons off longitudinally polarized protons. The analysing power in the $\sin \phi$ moment of the cross section is $0.019 \pm 0.007$ (stat.) \pm 0.003 (syst.). This result is compared to single-spin asymmetries for charged pion production measured in the same kinematic range. The $\pi^{0}$ asymmetry is of the same size as the $\pi^{+}$asymmetry and shows a similar dependence on the relevant kinematic variables. The asymmetry is described by a phenomenological calculation based on a fragmentation function that represents sensitivity to the transverse polarization of the struck quark.
\end{abstract}

PACS numbers: 13.87.Fh, 13.60.-r, 13.88.+e, 14.20.Dh

Semi-inclusive pion production in polarized deepinelastic scattering is a powerful tool for investigating the spin structure of the nucleon and providing information about the spin-dependent parton distribution and fragmentation functions. The recent observation of a significant azimuthal asymmetry for semi-inclusive $\pi^{+}$ production in deep-inelastic scattering (DIS) of unpolarized positrons off longitudinally polarized protons [1] has revealed effects of quark distribution and fragmentation functions that describe the transverse polarization of quarks. In particular, the occurrence of single-spin asymmetries, where only the target is polarized, offers access to the fundamental but still unmeasured chiralodd transversity distribution functions [2, 3] through the so-called Collins effect [4 [7]. This effect involves a chiralodd fragmentation function, the Collins function, that describes the fragmentation of a transversely polarized quark into an unpolarized hadron and is discussed in recent experimental [1,8] and theoretical publications [9 19] on this subject.

Single-spin asymmetries in pion production have already been measured in proton-proton scattering experiments [20], where the $\pi^{0}$ asymmetry is found to be similar to the $\pi^{+}$one, but with opposite sign as compared with the $\pi^{-}$one. This isospin dependence could be understood 21] in terms of the difference between the unpolarized cross sections for $\pi^{+}$and $\pi^{-}$production. However, these asymmetries from proton-proton scattering may also arise from initial-state interactions, which are negligible for semi-inclusive lepton-nucleon scattering processes. In the latter case any single-spin asymmetry can originate only from a spin dependence in the fragmentation of a polarized quark. In fact such a dependence is assumed in [15, 16,22] where a sizeable $\pi^{0}$ single-spin asymmetry is predicted for lepton-nucleon scattering.

This paper reports the first observation of a single-spin azimuthal asymmetry in semi-inclusive neutral pion electroproduction. The relevant kinematic variables of this process in the target rest frame are the spacelike squared four-momentum $Q^{2}$ of the exchanged virtual photon with energy $\nu$, the pion fractional energy $z=E_{\pi} / \nu$, the pion transverse momentum $P_{\perp}$, and the azimuthal angle $\phi$ of the pion around the virtual-photon axis. Here $E_{\pi}$ is the pion energy, $P_{\perp}$ is defined with respect to the virtualphoton direction and $\phi$ is defined relative to the lepton scattering plane. The fractional energy transferred to the proton is given by $y=\nu / E$ and the Bjorken scaling variable is defined as $x=Q^{2} / 2 M \nu$, where $E$ is the lepton beam energy and $M$ is the proton mass.

The data were collected in 1996 and 1997 using a longitudinally polarized hydrogen gas target in the $27.57 \mathrm{GeV}$ HERA positron storage ring at DESY. The average target polarization was 0.86 with a fractional uncertainty of $5 \%$. The scattered positron and the decay photons from the $\pi^{0}$ were detected by the HERMES spectrometer [23]. Positrons were distinguished from hadrons with an average efficiency of $99 \%$ at a hadron contamination of less than $1 \%$ using the information from an electromagnetic calorimeter, a transition radiation detector, a preshower scintillator detector, and a threshold Cerenkov detector. The kinematic requirements imposed on the scattered positrons were $Q^{2}>1 \mathrm{GeV}^{2}, 0.023<x<0.4$, $0.2<y<0.85$, and an invariant mass squared of the initial photon-nucleon system $W^{2}>4 \mathrm{GeV}^{2}$.

Neutral pion identification was provided by the detection of the two photon clusters originating from the $\pi^{0}$ decay in the electromagnetic calorimeter [24], each with a minimum energy deposition of $1.0 \mathrm{GeV}$ and without a corresponding charged track. The reconstructed photon-pair invariant mass $m_{\gamma \gamma}$ shows a clear $\pi^{0}$ mass peak with a mass resolution of about $0.012 \mathrm{GeV}$. Neutral pions were selected within the invariant mass range of $0.10<m_{\gamma \gamma}<0.17 \mathrm{GeV}$ where background contributions from uncorrelated photons typically amount to $20 \%$. An upper limit of $z<0.7$ was used in order to minimise acceptance effects and to suppress possible contributions of exclusive processes. The requirement $P_{\perp}>0.05 \mathrm{GeV}$ ensures an accurate measurement of the azimuthal angle $\phi$. 
The analysing powers for unpolarized (U) beam and longitudinally (L) polarized target are evaluated as

$$
A_{\mathrm{UL}}^{W}=\frac{\frac{L^{\rightarrow}}{L_{\overrightarrow{\mathrm{P}}}} \sum_{i=1}^{N \rightarrow} W\left(\phi_{i}\right)-\frac{L^{\leftarrow}}{L_{\mathrm{P}}^{\leftarrow}} \sum_{i=1}^{N^{\leftarrow}} W\left(\phi_{i}^{\leftarrow}\right)}{\frac{1}{2}\left[N \rightarrow+N^{\leftarrow}\right]},
$$

using the weighting functions $W(\phi)=\sin \phi$ and $W(\phi)=$ $\sin 2 \phi$. Here, the superscripts $\rightarrow$ and $\leftarrow$ denote opposite target helicity states. Each summation runs over the number $N^{\rightarrow(\leftarrow)}$ of pions selected for each target helicity state and is multiplied with the dead-time corrected luminosities $L^{\rightarrow(\leftarrow)}$ and $L_{\mathrm{P}}^{\rightarrow(\leftarrow)}$, the latter being weighted by the target polarization magnitude. The analysing powers were determined by integrating over the spectrometer acceptance in the kinematic variable $y$ with a mean value of 0.57 . Small corrections were applied for crosscontamination between the $\sin \phi$ and $\sin 2 \phi$ moments due to the spectrometer acceptance, based on a Monte Carlo simulation. Aside from this, the results have been shown to be little affected by the limited acceptance of the HERMES spectrometer. A background contribution from uncorrelated photons was estimated by varying the window in the reconstructed invariant mass of the photon pair. It was found to be negligible within the statistical accuracy of the data and was taken into account in the systematic error. The primary contributions to the systematic uncertainty arise from the target polarization and from the acceptance corrections. Radiative effects on the unpolarized cross section were evaluated and a contribution to the asymmetry of less then $0.1 \%$ averaged over the full acceptance was found [25].

The analysing power in the azimuthal $\sin \phi$ moment of the $\pi^{0}$ production cross section, averaged over $z, x$, and $P_{\perp}$ with mean values of $0.48,0.09$, and $0.44 \mathrm{GeV}$, respectively, is $0.019 \pm 0.007$ (stat.) \pm 0.003 (syst.). This result is consistent with the $\pi^{+}$measurements for which an analysing power of $0.022 \pm 0.005$ (stat.) \pm 0.003 (syst.) was reported [1]. The observed azimuthal asymmetry implies a substantial magnitude for the Collins fragmentation function $H_{1}^{\perp}$. The analysing power in the $\sin 2 \phi$ moment for the same process, calculated using Eq. (1), is consistent with zero within the statistical uncertainty: $0.006 \pm 0.007$ (stat.) \pm 0.003 (syst.). This is expected from predictions for the ratio of $A_{\mathrm{UL}}^{\sin 2 \phi}$ to $A_{\mathrm{UL}}^{\sin \phi}$ [11], which are small in the valence region for the specific kinematic range of relatively low $Q^{2}$ and moderate $P_{\perp}$ accessible at HERMES.

In Fig. 1 the analysing power $A_{\mathrm{UL}}^{\sin \phi}$ is shown as a function of the pion fractional energy $z$, the Bjorken scaling variable $x$, and the pion transverse momentum $P_{\perp}$, after averaging over the other two kinematic variables. Also shown are results for charged pions [1] obtained in the same kinematic range. The $\pi^{0}$ and $\pi^{+}$asymmetries exhibit a similar behaviour in all kinematic variables. The $z$ dependence of the $\pi^{0}$ asymmetry is consistent with the monotonic increase of the $\pi^{+}$asymmetry. The increase of $A_{\mathrm{UL}}^{\mathrm{sin} \phi}$ with increasing $x$ suggests that single-spin asymmetries are associated with valence quark contributions.

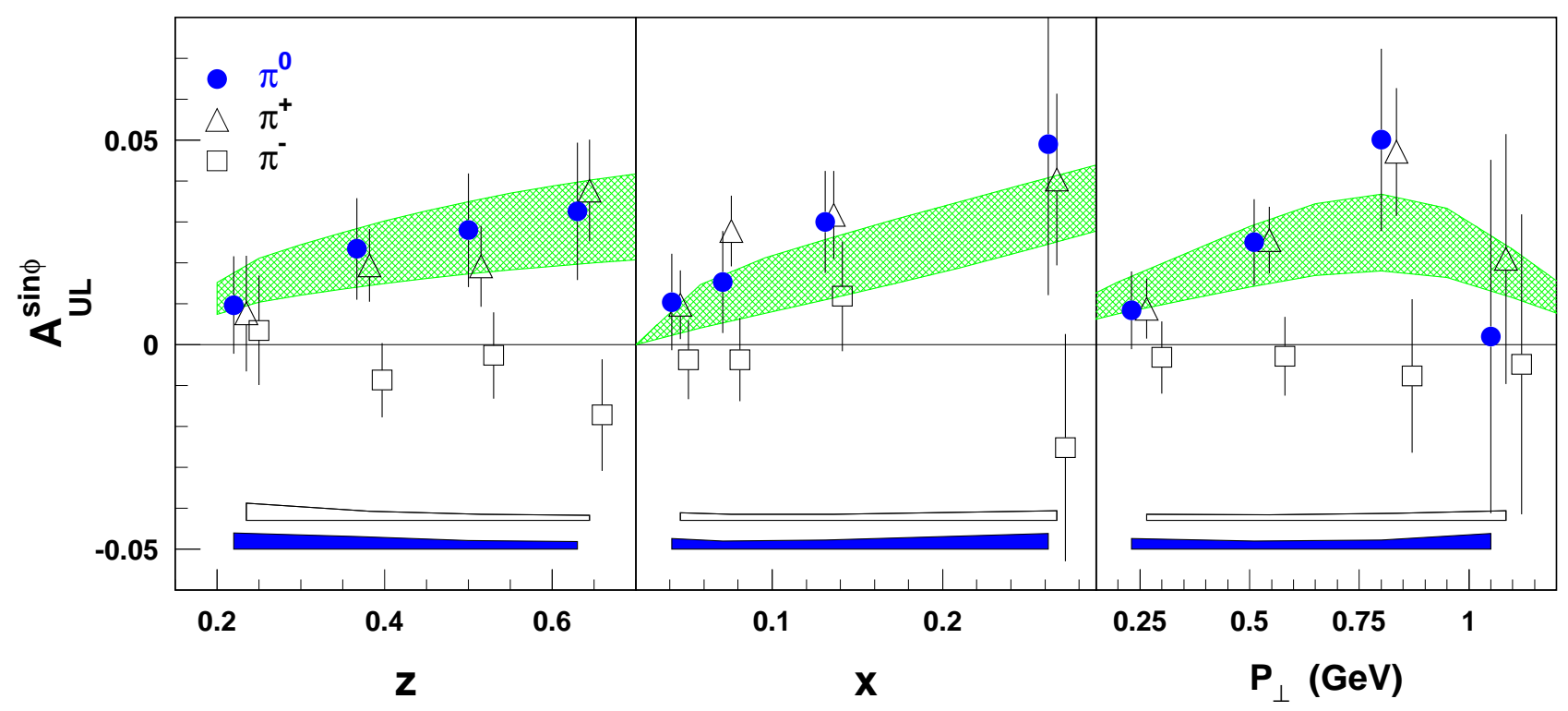

FIG. 1. Analysing power in the $\sin \phi$ moment for $\pi^{0}$ (circles) compared to previous results $\| 1$ for $\pi^{+}$(triangles) and $\pi^{-}$ (squares) as a function of the pion fractional energy $z$, the Bjorken variable $x$, and of the pion transverse momentum $P_{\perp}$. Error bars include the statistical uncertainties only. The filled and open bands at the bottom of the panels represent the systematic uncertainties for neutral and charged pions, respectively. The data for charged pion production are slightly shifted in $z, x$, and $P_{\perp}$ for clarity. The shaded areas show a range of predictions of a model calculation [10,17 applied to the case of $\pi^{0}$ electroproduction (see text). 
The dependence on $P_{\perp}$ can be related to the dominant kinematic role of the intrinsic transverse momentum of the quark, if $P_{\perp}$ remains below the typical hadronic mass of about $1 \mathrm{GeV}$ [1]. Also shown in Fig. 11 are the predictions of a model calculation 10,17] for the $\pi^{0}$ single-spin azimuthal asymmetry using isospin and charge conjugation invariance 15]. In the case of longitudinally polarized nucleons, two distribution functions enter the calculation. One, $h_{1 \mathrm{~L}}^{\perp}$, is twist-2 and describes the quark transverse spin distribution in a longitudinally polarized nucleon, while $h_{\mathrm{L}}$ includes an interaction dependent twist-3 part. Both are related to the twist-2 distribution function $h_{1}$, called transversity, that describes the quark transverse spin distribution in a transversely polarized nucleon, by $h_{\mathrm{L}}(x)=h_{1}(x)-\frac{\mathrm{d}}{\mathrm{dx}} h_{1 \mathrm{~L}}^{\perp(1)}(x)$ [6], where $h_{1 \mathrm{~L}}^{\perp(1)}$ is the $k_{\mathrm{T}}^{2}$-moment of $h_{1 \mathrm{~L}}^{\perp}$ over the intrinsic quark transverse momentum $k_{\mathrm{T}}$. Assuming a vanishing $h_{1 \mathrm{~L}}^{\perp(1)}$ 17, the number of unknown distribution functions can be reduced to one: $h_{\mathrm{L}} \simeq h_{1}$. The range of predictions, shown in Fig. 1, is obtained by varying $h_{1}$ between the two assumptions $h_{1}=g_{1}$ (non-relativistic limit) and $h_{1}=\left(f_{1}+g_{1}\right) / 2$ (Soffer inequality), with the usual polarized and unpolarized distribution functions $g_{1}$ and $f_{1}$, respectively. In both cases a simple parameterisation for the spin-dependent time-reversal-odd fragmentation function $H_{1}^{\perp}$ was adopted. The predictions are consistent with the measured $\pi^{0}$ azimuthal asymmetries and describe the dependences on the kinematic variables. These new $\pi^{0}$ data provide additional information also for other phenomenological approaches 12 14,19.

In summary, a single-spin azimuthal asymmetry for $\pi^{0}$ production has been measured in semi-inclusive deepinelastic lepton scattering off a longitudinally polarized proton target. The dependence of this asymmetry on the kinematic variables $x, z$, and $P_{\perp}$ has been investigated. The results are similar to the previously measured azimuthal asymmetry for $\pi^{+}$electroproduction, while the $\pi^{-}$asymmetry was consistent with zero. This finding can be well described by a model calculation where the asymmetry is interpreted as the effect of the convolution of a chiral-odd distribution function and a time-odd fragmentation function. The observed single-spin azimuthal asymmetries for neutral and charged pion electroproduction are consistent with the assumed $u$-quark dominance in both distribution and fragmentation functions. These results provide evidence in support of the existence of non-zero chiral-odd structures that describe the transverse polarization of quarks. New data are expected from future HERMES measurements on a transversely polarized target, which will give direct access to the transversity [26].

We thank M. Anselmino, R.L. Jaffe, A.M. Kotzinian, and P.J. Mulders for many interesting discussions on this subject. We gratefully acknowledge the DESY management for its support, the staffs at DESY, and the collaborating institutions for their significant effort, and our funding agencies for financial support. Additional support for this work was provided by the DAAD and INTAS, HCM, TMR network contributions from the European Community, and the U.S Department of Energy.

[1] HERMES Collaboration, A. Airapetian et al., Phys. Rev. Lett. 84, 4047 (2000).

[2] J.D. Ralston and D.E. Soper, Nucl. Phys. B 152, 109 (1979).

[3] R.L. Jaffe and X. Ji, Phys. Rev. Lett. 67, 552 (1991) and Nucl. Phys. B 375, 527 (1992).

[4] J. Collins, Nucl. Phys. B 396, 161 (1993).

[5] A.M. Kotzinian, Nucl. Phys. B 441, 234 (1995).

[6] P.J. Mulders and R.D. Tangermann, Nucl. Phys. B 461, 197 (1996).

[7] R.L. Jaffe, X. Jin, and J. Tang, Phys. Rev. Lett. 80, 1166 (1998).

[8] A. Bravar for the SMC Collaboration, Nucl. Phys. B(Proc. Suppl.) 79, 520 (1999).

[9] P.J. Mulders, Nucl. Phys. A 622, 239c (1997).

[10] K.A. Oganessyan, H.R. Avakian, N. Bianchi, and A.M. Kotzinian, hep-ph/9808368.

[11] A.M. Kotzinian, K.A. Oganessyan, H.R. Avakian, and E. De Sanctis, Nucl. Phys. A 666\&667, 290c (2000).

[12] D. Boer, RIKEN Rev. 28, 26 (2000); hep-ph/9912311.

[13] A.V. Efremov, K. Goeke, M.V. Polyakov, and D. Urbano, Phys. Lett. B 478, 94 (2000).

[14] M. Boglione and P.J. Mulders, Phys. Lett. B 478, 114 (2000).

[15] M. Anselmino and F. Murgia, Phys. Lett. B 483, 74 (2000).

[16] R.L. Jaffe, hep-ph/0008038 and hep-ph/0102281.

[17] E. De Sanctis, W.-D. Nowak, and K.A. Oganessyan, Phys. Lett. B 483, 69 (2000).

[18] A. Schäfer and O.V. Teryaev, Phys. Rev. D 61, 077903 (2000).

[19] B. Ma, I. Schmidt, and J. Yang, Phys. Rev. D 63, 037501 (2001).

[20] B.E. Bonner et al., Phys. Rev. D 41, 13 (1990); D.L. Adams et al., Phys. Lett. B 264, 462 (1991); A. Bravar et al., Phys. Rev. Lett. 77, 2626 (1996).

[21] M. Anselmino, M. Boglione, and F. Murgia, Phys. Lett. B 362, 164 (1995).

[22] C. Boros, Z. Liang, and R. Rittel, J. Phys. G: Nucl. Part. Phys. 24, 75 (1998).

[23] HERMES Collaboration, K. Ackerstaff et al., Nucl. Instrum. Methods A 417, 230 (1998).

[24] H. Avakian et al., Nucl. Instrum. Methods A 417, 69 (1998).

[25] I. Akushevich, N. Shumeiko, and A. Soroko, Eur. Phys. J. C 10, 681 (1999).

[26] V. Korotkov, W.-D. Nowak, and K. Oganessyan, Eur. Phys. J. C 18, 639 (2001). 